\title{
Single-shot beam size measurements using visible-light interferometry at CESR
}

S.T. Wang ${ }^{a, *}$, R. Holtzapple ${ }^{b}$, D.L. Rubin ${ }^{a}$

${ }^{a}$ Cornell Laboratory for Accelerator-based Science and Education, Cornell University, Ithaca, New York, 14853

${ }^{\text {b} P h y s i c s ~ D e p a r t m e n t, ~ C a l i f o r n i a ~ P o l y t e c h n i c ~ S t a t e ~ U n i v e r s i t y, ~ S a n ~ L u i s ~ O b i s p o, ~ C A, ~} 93407$

*Corresponding author: 218 Wilson Lab, Ithaca, NY 14853. E-mail: sw565@cornell.edu Tel: 607-2558777. Fax: 607-255-8062

\section{ABSTRACT}

A new primary mirror for a visible-light beam size monitor (VBSM) was designed and installed in the Cornell Electron-Positron Storage Ring (CESR). The vertical angular acceptance of the mirror was doubled to allow double-slit interferometry with large slit separation (>12 $\mathrm{mm}$ ). In addition, the diffraction associated with the first generation mirror has been eliminated. The resolution of the vertical beam size measurements has been dramatically improved but is ultimately limited by the beam motion. Two fast-response detectors, a Photomultiplier Tube (PMT) array and a gated camera, were employed to study the beam motion. The advantages and limitations of both devices are discussed in this paper. The gated camera was also used to measure single-shot beam width and motion of each bunch in a multi-bunch train. We measured significantly more horizontal motion of electron as compared to positron bunch trains in otherwise identical machine condition. This difference may be a signature for the difference between electron cloud build-up for positron bunch trains versus ions effects characteristic of electron bunch trains.

Keywords: Interferometer; Synchrotron radiation; Beam size monitor; Gated camera; Bunch-bybunch 


\section{Introduction}

Synchrotron radiation (SR) from bending magnets, mainly visible light and $\mathrm{x}$-rays, in circular accelerators has been widely used to monitor transverse and longitudinal beam profiles. Direct imaging utilizing the visible light spectrum is the main method to acquire the beam dimensions when the beam sizes are relatively large $(>100 \mu \mathrm{m})$. However, modern low emittance storage rings operating as sources of $\mathrm{x}$-rays or as damping rings for linear colliders have much smaller beam sizes ( $\sim 10 \mu \mathrm{m})$ where more sophisticated techniques are required for measuring beam dimensions. To overcome the diffraction limit, beam size monitors using $x$-rays have been developed in many accelerator facilities [1-4]. Alternatively, the SR interferometer first applied by Mitsuhashi [5-7] is by now a well-developed technique for accurately measuring transverse beam sizes of less than $300 \mu \mathrm{m}$ using visible light [6]. Another approach to measure small beam sizes less than $30 \mu \mathrm{m}$ is to image the vertical $(\pi)$ polarization component of the visible SR light. This technique has been successfully implemented at the Swiss Light Source to measure the vertical beam size less than $10 \mu \mathrm{m}$ [8].

Most visible light transverse beam profile monitors use conventional CCD cameras to measure beam dimensions, whether by direct imaging, an interferometer, or $\pi$-polarization methods. CCD cameras are intensity-integrated devices that collect light over a period of order 10 1000 ms. Thus, the collected signals are the integrated light from all bunches in the storage ring over many turns. For example, the CESR revolution period is about $2.56 \mu \mathrm{s}$, and typical exposure time to obtain an interference pattern for a single bunch of $0.75 \mathrm{~mA}$ is about $50 \mathrm{~ms}$, which is about 20 thousand turns in CESR. Therefore, when there is transverse motion, either turn-by-turn or bunch-by-bunch, the profile measurements using conventional CCD camera will be compromised.

To eliminate the effect of pulse-to-pulse transverse beam motion in the study of multi-bunch beam dynamics along a bunch train, fast detectors, such as the x-ray beam size monitor (xBSM) and the fast response Photomultiplier Tube (PMT) array, with response time less than a few nanoseconds are required. An x-ray beam size monitor (XBSM) was built to measure the vertical beam size blowup along a CESR positron train due to electron cloud effect [4]. This monitor employs a fast-response diode array and fast read-out electronics that resolve turn-by-turn, bunch-by-bunch beam sizes along a 4ns spaced bunch train. In addition, a fast response PMT array, using a visible synchrotron radiation from the CESR beam, is used as a visible light beam size monitor (VBSM), which has bunchby-bunch and turn-by-turn capability, to measure both horizontal and vertical beam sizes [9] in CESR. These two devices utilize one-dimensional array detectors for single axis beam size measurements.

Fast-gated cameras, with a variable gate that can resolve 4 ns spaced bunches, have been utilized to quantify turn-by-turn beam profiles from an injected beam [10-12] as well as for a stored beam [1315]. The bunch-by-bunch capability has also been demonstrated [16-19]. All these measurements are based on direct imaging profiles rather than interferometric measurements.

In this paper, we present the recent upgrades to the beam size monitor instrumentation and the development of the beam profile monitors to measure the transverse beam dynamics of multibunch trains in CESR. A new primary mirror with increased vertical acceptance was installed in CESR recently, for compatibility with the widely spaced slits $(>12 \mathrm{~mm})$ necessary to measure smaller transverse beam sizes using the SR interferometer. The larger acceptance of the mirror also eliminated the diffraction observed in imaging the $\pi$-polarization profile using the first generation 
mirror. With these dramatic improvements in the sensitivity of the vertical beam size measurements the resolution is now limited by pulse-to-pulse transverse beam motion. The effect of the jitter was quantified using two different fast-response detectors, a PMT array and a gated camera. Advantages and limitations of both devices will be discussed in this paper. The fast-gated camera was utilized to record bunch-by-bunch interferometry along a train to quantify the bunch dependence of the horizontal beam sizes under various operating conditions of CESR, and for the first time, we obtained the horizontal interference patterns of each bunch in a 30-bunch train for both positrons and electrons. From these measurements, we determined that the beam dynamics are quite different for trains of positron and electron.

\section{Experimental methods}

\subsection{Experimental Setup}

CESR is a storage ring for both electron and positron beams with mirror symmetry about the northsouth diameter. Two vBSMs are installed symmetrically about the north-south axis, at east and west end of the north area straight, for monitoring electron and positron beams, respectively. The vBSM primary mirrors are located symmetrically at opposite ends of the north area straight. Figure 1a shows the schematic layout of the experimental facilities of the VBSMs. The visible SR from a soft bending magnet is reflected by the primary Beryllium mirror that is mounted inside the vacuum chamber. With the installation of a larger mirror, the vertical acceptance was doubled to $2.5 \times 4.4$ mrad $(\mathrm{H} \times \mathrm{V})$. The larger vertical acceptance serves to reduce diffraction effects and to accommodate more widely spaced double slits. Through an adjustable iris, SR photons reach the double slits and a focusing lens $(f=5 \mathrm{~m})$, which is $6 \mathrm{~m}$ from the source point. Multiple mirrors are used to guide the $S R$ light to the optical table located $27 \mathrm{~m}$ from the source point. The SR light from both electrons and positrons is reflected by a large 6 "x 12 " mirror into the experimental hall outside the tunnel of north ring. On the optical table, the SR passes through a lens $(f=1 \mathrm{~m})$, a polarizer, a $500-\mathrm{nm}$ narrow band pass filter. Finally, the light is imaged on either a conventional CCD or a fast-gated camera.

The double slits are mounted on a translation stage so that they can be moved in or out of the light path to allow for direct imaging or interferometry measurements of the beam size. For interferometry of the horizontal beam size, one set of vertically aligned double slits with fixed slit separation (e.g. $D=2.0 \mathrm{~mm}$ ) is moved into the beam path. Alternatively, sets of double slits can be easily installed for either horizontal, vertical, or simultaneous horizontal and vertical beam size measurements. Details of our vBSM setup can be found in [20].

In our experiments, a single species was stored in CESR. Since the measurements acquired for beams with a single electron or positron bunch are similar, unless explicitly indicated, only the results for electron beam size measurements are discussed. Measurements with beam energies of $2.1 \mathrm{GeV}$ and $1.8 \mathrm{GeV}$ are explored in our experiments. However, most measurements are done at $2.1 \mathrm{GeV}$. The beam parameters for the two different beam energies at the vBSM source point are listed in Table 1.

\subsection{Measurement theory}

The principles of the beam size interferometer with double slits have been discussed in many papers $[5,6]$. Basically, the instrument measures the degree of coherence of the polarized quasi- 
monochromatic light. When the light intensities at the two slits are the same, the interference pattern at the detector plane can be written as $[7,21]$

$$
I(x)=I_{0}\left[\operatorname{sinc}\left(\frac{2 \pi a}{\lambda R} x+\phi\right)\right]^{2}\left(1+|\gamma| \cos \left(\frac{2 \pi D}{\lambda R} x+\psi\right)\right)
$$

where $I_{0}$ is the light intensity through the slits, $\lambda$ is the wavelength, $a$ is the half width of the slit, and $D$ is the slit separation. $R$ denotes the distance from the back principal point of the lens to the detector. Since a second lens is used in our system (Fig. 1a), $R$ is slightly shorter than the actual distance. $\phi$ and $\psi$ are phase shifts. $\gamma$ denotes the spatial coherence (or visibility). The spatial coherence can evidently be extracted from the measured intensity distribution. For a Gaussianshape beam profile in the horizontal direction $(x)$, its width $\sigma_{x}$ is related to spatial coherence $\gamma$ according to

$$
\sigma_{x}=\frac{\lambda L}{\pi D} \sqrt{\frac{1}{2} \ln \frac{1}{\gamma}}
$$

where $L$ is the distance from the source to the slits. By acquiring and fitting the interference pattern to obtain the spatial coherence $\gamma$, we obtain the beam size $\sigma_{x}$. Similarly, the vertical beam size $\sigma_{y}$ is obtained by acquiring the $\gamma$ in the vertical direction.

Another technique to measure the vertical beam size is from the $\pi$-polarized vertical beam profile. By extracting the valley-to-peak intensity ratio and comparing to the theoretical curve, we infer the vertical beam size $[8,20]$. This $\pi$-polarization method of measuring the vertical beam size is discussed in detail in ref [8].

\subsection{Primary Beryllium mirror}

The maximum angular acceptance is determined by the dimensions of the primary Be mirror (Fig. 1a). The vertical dimension of the old Be mirror is $12.5 \mathrm{~mm}$ (Fig. 1b), corresponding to angular acceptance of $2.2 \mathrm{mrad}$. This angular acceptance is sufficient to capture most of SR radiation because of small natural beam opening angle of the radiation. For example, the natural opening angle of $\lambda=500 \mathrm{~nm}$ SR light is $0.69 \mathrm{mrad}$. However, the old mirror limits the maximum separation of double-slits for the interferometry measurement. As Eq. (2) shows, larger separation $D$ allows measurement of smaller size $\sigma_{x}$, for a given visibility $\gamma$. Thus, increasing $D$ will increase the measurement sensitivity to smaller beam sizes.

The upgraded primary Be mirror has a larger vertical dimension $(25 \mathrm{~mm})$, as shown in Fig. 1c. The image of the Be mirror illuminated by visible SR from a $0.75 \mathrm{~mA}$ positron bunch is shown in Fig. $2 \mathrm{a}$. The left circular edge indicates where the SR light is clipped by the circular extraction vacuum port. Fig. $2 \mathrm{~b}$ shows the image of a set of double slits with a separation of $14 \mathrm{~mm}$ with the upgraded Be mirror, demonstrating that the larger mirror acceptance is compatible with widely spaced slits. As will be discussed in the following, three sets of double slits $(D=10,14,18 \mathrm{~mm})$ were used to measure the vertical beam sizes (Fig. 4).

\subsection{Fast-response detectors}


To quantify the degradation of our resolution due to transverse beam motion, we used two different fast beam size detectors. The first is a multi-anode PMT manufactured by Hamamatsu [22]. This PMT consists of a 32-channel linear array with $1 \mathrm{~mm}$ channel pitch and $7 \mathrm{~mm}$ width. It has a high speed response with a sub-nanosecond rise time and can provide turn-by-turn single bunch measurements of the vertical beam size and motion in a train with $4 \mathrm{~ns}$ spacing. The electronic circuits and boards were custom built to take advantage of the high speed of the device. Custom Matlab code was developed for control and data acquisition of the PMT.

The second detector is a gated CCD camera with $1024 \times 1024$ pixels, a PI-MAX4 camera from Princeton Instruments [23]. Its minimum gate width is $\sim 3 \mathrm{~ns}$ which allows resolution of an image of a single bunch in a multi-bunch train with $4 \mathrm{~ns}$ or $14 \mathrm{~ns}$ spacing. The camera is triggered on a single bunch by using a $60-\mathrm{Hz}$ timing trigger derived from a clock synchronized with the CESR turn clock. A fine trigger delay of $80 \mathrm{ps}$ is used to place the beam signal at the center of the camera gate. In order to measure beam size of a specific bunch in a multi-bunch train, the trigger delay needs to be changed accordingly. The procedure of stepping the trigger delay along a bunch train, and recording bunch images sequentially, is managed by a custom LabVIEW program through an EPICS interface.

\section{Results and analysis}

\subsection{Vertically polarized beam profile}

A comparison of the component of the vertically polarized beam profile of a single positron bunch at $0.75 \mathrm{~mA}$ is shown in Figs. $3 a$ and $3 \mathrm{c}$ with the old and new Be mirror respectively. Diffraction peaks are present around the two main vertical peaks in the image (Fig. 3a) and intensity profile (Fig. 3b) with the old mirror, and have been eliminated with the new mirror (Figs. $3 \mathrm{c}$ and $3 \mathrm{~d}$ ). The vertical beam size using vertically polarized synchrotron radiation is computed from the ratio of the valleyto-peak of the intensity profile and diffraction contaminates the profile. When the vertical dimension of the Be mirror was doubled, the diffraction peaks disappeared (Fig. 3d) thus eliminating the systematic error associated with diffraction.

\subsection{Vertical beam size measurements}

Another benefit of the new Be mirror is that horizontally aligned double slits with larger separation $(>12 \mathrm{~mm}$ ) can be used in the interferometry measurements of the vertical beam size. Figure 4 shows three CCD images taken with three sets of double slits with spacing of $D=10,14,18 \mathrm{~mm}$. The vertical intensity profiles (Figs. 4a-c) are $\chi^{2}$ fit to Eq. (1) to determine the images' visibility $\gamma$, which is plotted as a function of $D$ in Fig. $4 \mathrm{~d}$. The error bars shown in Fig. $4 \mathrm{~d}$ are the standard deviation from many measurements over a time frame of one minute. Since the SR light intensity through the $18 \mathrm{~mm}$ double slits is lower than the other slit spacing, the exposure time of CCD camera is increased in order to acquire enough light to establish the interference pattern. Therefore, the measurements with $18 \mathrm{~mm}$ double slits are susceptible to a larger systematic error in the measurement of the vertical beam size due to transverse beam motion or system vibration. From the $\chi^{2}$ fit of $\gamma$ vs $D$ using Eq. (2), we found the vertical beam size of a single $2.1 \mathrm{GeV}$ positron bunch at $0.6 \mathrm{~mA}$ is about 45.8 $\mu \mathrm{m}$.

A comparison between the two methods of measuring the vertical beam size using visible light (interferometer and vertically polarized light) was made with the x-ray Beam Size Monitor (XBSM) as 
the current in the single bunch decayed from $5 \mathrm{~mA}$ to $0.25 \mathrm{~mA}$ at $2.1 \mathrm{GeV}$. The data are shown in Fig. 5. The red circles are the vertical beam sizes at the XBSM source point. The blue crosses in Fig. $5 \mathrm{a}$ and $5 \mathrm{~b}$ are the vertical beam sizes at the $\mathrm{VBSM}$ source point using interferometer and $\pi$-polarization methods, respectively. A 14-mm spaced double slit was used for the interferometer measurements. The vBSM data have been scaled by the beta-function-ratio for the two different devices shown in Eq. (3).

$$
\sqrt{\frac{\beta_{x B S M}}{\beta_{v B S M}}}=\sqrt{\frac{40.5}{20.3}}=1.41
$$

It is evident from Fig. 5 that the vertical beam size measurements with the VBSM have the same overall current dependence as the results from the XBSM. The vBSM data exhibit a large scatter. This large scatter is mainly due to turn-by-turn transverse beam motion. The XBSM measures beam size on a turn-by-turn basis and is therefore immune to the effects of the beam motion. Another observation is that the vertical size $\sigma_{y}$ measured by the $\pi$-polarization device produces a consistently smaller beam size than the interferometer. This can again be attributed to transverse beam motion as the exposure time of CCD camera required to obtain the $\pi$-polarized beam profile is significantly less than to acquire the interference pattern. For example, for a $0.75 \mathrm{~mA}$ single bunch, the exposure time to acquire the interference pattern with $14 \mathrm{~mm}$ double slit is about $100 \mathrm{~ms}$ as compared to only $10 \mathrm{~ms}$ to obtain the $\pi$-polarized beam profile.

To determine the minimum measurable vertical beam size, variable closed local beta bumps $\left(\beta_{y}\right)$ were constructed at the VBSM source point, allowing the adjustment of beam size at the source point without affecting global lattice properties. By varying the local bumps, we acquired the vertical beam size $\sigma_{y}$ using the two vBSM devices with a single positron bunch of $0.75 \mathrm{~mA}$ at beam energy $1.8 \mathrm{GeV}$. The results are shown in Fig. 6 and it is evident that the $\pi$-polarization method consistently measures, by about $3 \mu \mathrm{m}$, a smaller vertical beam size than the interferometer. At the smallest beta $\beta_{y} \sim 5 \mathrm{~m}$, the vertical beam size measured by the interferometer is $20.7 \mu \mathrm{m}$ and $17.0 \mu \mathrm{m}$ by the $\pi$ polarization device. However, the $\pi$-polarization method also shows larger beam size variation at each data point. This is likely due to variation of imbalanced peak intensity between two lobes in $\pi$ polarized beam profile. The measured vertical beam size $\sigma_{y}$ is related to the vertical emittance $\varepsilon_{y}$, Twiss parameters $\beta_{y}$, and some unknown source error $\sigma_{y 0}$ as shown in Eq. 4.

$$
\sigma_{y}^{2}=\beta_{y} \varepsilon_{y}+\sigma_{y 0}^{2}
$$

If we fit $\sigma_{y}$ as a function of $\beta_{y}$ using Eq. (4) assuming $\sigma_{y 0}$ does not depend on $\beta_{y}$, we can determine the vertical emittance and the zero current (minimum) vertical beam size. The best fit to the data is plotted as red lines in Fig. 6 . The fit yields $\varepsilon_{y}=17.0 \mathrm{pm}$-rad and $\sigma_{y 0}=19.2 \mu \mathrm{m}$ from interferometer and gives $\varepsilon_{y}=17.0 \mathrm{pm}$-rad and $\sigma_{y 0}=14.6 \mu \mathrm{m}$ from $\pi$-polarization method. The extracted vertical emittance from both methods is 17.0 pm-rad, which agrees well with the measured emittance by XBSM [4]. Besides the vertical emittance, the contributions to the vertical beam size, $\sigma_{y 0}$, are the vertical dispersion at the source point, transverse beam motion, or some other measurement error due to vibration of the primary mirror and other optical elements in the long optical transport line. The measured RMS dispersion around the CESR ring is less than $12 \mathrm{~mm}$. Since the beam energy spread is only $0.077 \%$, contribution to vertical beam size due to dispersion is negligibly small. Contribution to vertical beam size error $\left(\sigma_{y 0}\right)$ due to transverse motion will be discussed in the next section. 


\subsection{Transverse Beam Motion}

The transverse beam size as measured by the CCD camera includes transverse beam motion. To quantify the transverse beam motion at the VBSM source point, we took turn-by-turn beam position data at a Beam Position Monitor (BPM) close to the VBSM source point. Analysis of the data shows an approximately $30 \mu \mathrm{m}$ turn-by-turn beam motion in the vertical direction. For interferometry, this beam motion generates a phase shift in the interference pattern that will reduce the visibility and result in an overestimate of the beam size. The phase error $\Delta \psi$ is related to the orbit offset $\Delta y$ by [24]

$$
\Delta \psi=\frac{2 \pi}{\lambda} \frac{D}{L} \Delta y
$$

The estimated average visibility for many turns is given by [20]

$$
\bar{\gamma}=\operatorname{sinc}(\Delta \psi) \gamma
$$

From Eq. (6) and assuming a $30 \mu \mathrm{m}$ beam motion, we estimate the measured vertical beam size $\sigma_{m}$ as function of the actual beam size $\sigma_{y}$ for three different slits separations, as shown in Fig. 7a. The error $\Delta \sigma_{y}=\sigma_{m}-\sigma_{y}$ is plotted in Fig. 7b. As we see from these plots, the error due to beam motion does not depend on the separation of the double slits. When the actual beam size becomes smaller, the beam motion causes the measured beam size to plateau so that the measurement error becomes larger.

Knowing the beam motion, we can extract the actual beam size $\sigma_{y}$ from Fig. 7a, however, for small beam sizes, the measured beam size $\sigma_{m}$ will be at the plateau region. A small variation in $\sigma_{m}$ will result in large change in $\sigma_{y}$. As a result, this method yields large uncertainty in $\sigma_{y}$. Another approach is to use fast response detectors that measure the turn-by-turn beam size, which removes the beam motion from the measurement completely.

\subsection{Single Bunch Beam Size Detectors}

Two visible light fast beam size detectors are used to quantify the single bunch transverse beam size in CESR. The first detector is a linear array PMT [9]. This detector demonstrated good performance for measurement of the beam size in a turn-by-turn bunch-by-bunch mode. However, due to the limited number of pixels (32 pixels) and electronic crosstalk between pixels caused, there exists a large systematic error in the horizontal beam size measurements with the interferometer [9]. Further development is needed to improve the PMT system to make it a useful detector for measuring beam size.

The second detector is a gated camera which is a two-dimensional CCD detector with a gate that can capture the light pulses in a window as short as 3 ns. During normal CESR operation, where the spacing between two adjacent bunches is either 4 or $14 \mathrm{~ns}$, the gated camera is capable of capturing the image of each bunch in a CESR train. Single bunch beam dynamics measurements using the gated camera are described in the next section.

\subsubsection{Single bunch measurements}

Before setting up the gated camera, a CCD camera was staged to align the light optics. Once aligned, a reference interference image was taken with the CCD camera. A vertically oriented pair of slits 
with spacing $D=2.0 \mathrm{~mm}$ was inserted for both the CCD and gated camera measurements. Figure 8a shows the reference image taken with a single positron bunch of $0.7 \mathrm{~mA}$ stored in CESR. The extracted horizontal profile from the interference image is displayed in Fig. 8d. Fitting the interference pattern with Eq. (1) resulted in a visibility of $\gamma=0.575$, which according to our set-up, gives a horizontal beam size of $252.4 \mu \mathrm{m}$, in good agreement with theoretical properties of the lattice [25].

The gated camera was then positioned at the image focusing plane, replacing the CCD camera. The camera trigger delay was adjusted to maximize the single bunch intensity with a gate width set to the minimum value of 3 ns to capture a single bunch. The camera's MCP (Multi Channel Plate) gain has a range of 1 to 100. For a single bunch of $0.75 \mathrm{~mA}$ in CESR, a gain of 20 was selected to avoid saturation. One hundred frames were captured and stored in a single image file. Figure $8 \mathrm{~b}$ displays a single image which demonstrates that the light from a single bunch at $0.75 \mathrm{~mA}$ is sufficient to produce an interference pattern. It should be noted that the extracted profile (Fig. 8e) is noisy compared to the bunch averaged profile captured by the CCD camera (Fig. 8d). Fitting to the profile yields a visibility of $\gamma=0.62$. Figure $8 \mathrm{c}$ is a single bunch image averaged over 100 frames, which provides a much cleaner image but with a somewhat smaller visibility of $\gamma=0.54$ (Fig. 8f). This reduction in visibility is expected because the averaged image includes transverse beam motion that reduces the effective visibility. By processing the 100 frames individually, the frame-by-frame fitting, the effect of the beam motion is eliminated, as can be seen in the Appendix video 1 where the averaged visibility over 100 frames fitting is $\gamma=0.58 \pm 0.03$, slightly higher than that obtained from CCD camera.

To quantify transverse beam motion in CESR, 100 single-shot images of a Gaussian beam by the gated camera were recorded in the first row of Appendix video 2. The $x$ and $y$ movement of the beam may be partially due to actual beam motion and partially due to system vibration. The horizontal and vertical profiles of each image are the image projection on the horizontal and vertical axes, respectively. They were fit to a Gaussian distribution to determine the vertical and horizontal beam size and centroid of each image. The results of the fits are displayed in Fig. 9, where Fig. 9a and $9 \mathrm{c}$ are the horizontal and vertical beam size, and Fig. $9 \mathrm{~b}$ and $9 \mathrm{~d}$ are the horizontal and vertical bunch centroids. The standard deviation of $x$ and $y$ centroids are 0.97 and 0.63 pixels, respectively. Since the pixel size of the gated camera is $13.3 \mu \mathrm{m}$ and the image magnification is 0.2 , we estimate the maximum horizontal and vertical beam motion at the vBSM source point to be 65 and $42 \mu \mathrm{m}$, respectively. It is worth nothing that these values include system vibration so that they are higher than the $30 \mu \mathrm{m}$ beam motion measured by turn-by-turn BPM.

The $\sigma_{x}$ and $\sigma_{y}$ determined by fitting the average image of 100 frames is 7.52 and 3.13 pixels, respectively. Both are larger, due to transverse beam motion, than the sizes obtained by averaging the fits of 100 single frames $\left(\sigma_{x}=7.44, \sigma_{y}=3.05\right.$ pixels). To eliminate transverse beam motion with image averaging, we sum the 100 frames based on the centroid of the beam profile where each image is shifted to the same beam centroid. The result can be seen in the third row of Appendix video 2 . In this row of this video, the displayed $n$th image is the averaged image of $n$ shifted frames (from 1 to $\mathrm{n}$ ). The corresponding horizontal and vertical profiles were fit to obtain the intermediate $\sigma_{x}, \sigma_{y}$, and centroids. From fitting the shifted averaged beam profile, both the horizontal and vertical beam sizes $\left(\sigma_{x, y}\right)$ (green lines in Fig. 9) are reduced compared to the direct summation (red lines in 
Fig. 9). The direct summation procedure can be seen in the second row of Appendix video 2 , which is similar as the shift-summation procedure but without shifting the centroid.

The same centroid-shift-sum method is also applied to the interference patterns (Appendix video 3). The visibility obtained from centroid-shifted-sum image is similar to that from the direct sum image. This is understandable because the beam motion in the horizontal plane causes a phase shift in the interference pattern but no change in the centroid [24]. Shifting the centroid does not eliminate the smearing of the phase shift.

\subsubsection{Multiple bunch measurements}

As part of an electron cloud beam dynamics experiment, a train of 30 positron bunches with 14-ns spacing was stored in CESR at beam energy $2.1 \mathrm{GeV}$. To measure the horizontal beam size of the 30 bunch train, the gated camera trigger delay was changed in $14 \mathrm{~ns}$ steps to capture all the bunches along the train. For each bunch, 100 single-shot frames were recorded and stored in one image file. It took approximately 2 minutes to acquire the data for all 30 bunches in the train.

Figure 10 displays the average of 100 interferometer images for bunches $1,2,8,16,24$, and 30 in a 30 bunch train of $0.75 \mathrm{~mA}$ per bunch. Fitting the interference profiles yields the horizontal beam sizes. From the results shown in Fig. 12 , it is evident that the horizontal beam size $\left(\sigma_{x}\right)$ starts to increase after bunch 16 and continues to increase along the train. In addition, we see a similar behaviour in the vertical beam size along the train from bunch-by-bunch vertical beam size $\left(\sigma_{y}\right)$ measurements from the XBSM [26]. The increase of both $\sigma_{x}$ and $\sigma_{y}$ is likely due to the electron cloud build-up along the train [26]. Frame-by-frame fitting of the interference pattern for each bunch has also been performed. The average visibility for each bunch shows the similar behaviour along the train as that from fitting the average interference of each bunch, confirming that apparent increase of transverse beam size along the train is not simply an effect of the beam motion.

For comparison, a train of 30 electron bunches was studied under similar accelerator conditions as for the train of positron bunches. Images bunch 1, 10, 13, 16, and 30 are displayed in Fig. 11. The single-shot images are on the left (a-e) and the corresponding averaged images over 100 frames are on the right ( $f-j)$. The single shot images from these five bunches are similar. However, the averaged images are very different. The images from bunch 1 and 30 display clear interference peaks while the peaks in the images of bunch 10,13, and 16 are smeared out. The smearing of the interference pattern is due to the horizontal beam motion as we can see clearly in the Appendix video 4.

For both the positron and electron experiments the bunch by bunch horizontal feedback was enabled. The vertical feedback was off. Interestingly, we find the bunch by bunch horizontal motion of electron beam (Appendix video 5) is larger than in the positron beam (Appendix video 6) and so the fit to the averaged interference pattern for electrons is not particularly informative. The bunchby-bunch electron beam sizes are obtained by fitting 100 interference images individually. We find that $\sigma_{x}$ does not change along the train as shown in Fig. 12. Under different machine conditions, for example with different vertical chromaticity, we do observe a slightly increasing $\sigma_{x}$ along with the beam motion. Further studies of beam dynamics using the gated camera are presently underway.

The electron beam motion is likely due to the presence of ions in the vacuum chamber [27]. At low pressure (approximately $1 \mathrm{nTr}$ ), the beam size along the bunch train is not affected by the ions, 
however, when the vacuum is degraded, we observe a horizontal beam size increase and motion that can attributed to ions [27]. In contrast, under similar machine conditions, the positron beam motion along the train remains small even as the beam size increases along the bunch train. These experiments demonstrate the difference in transverse beam dynamics for the two different species of bunch trains in CESR; for positrons, the transverse beam dynamics is determined by the presence of the electron cloud, for electrons, the transverse beam dynamics is determined by the presence of ions.

\section{Conclusion}

The primary Beryllium mirror was upgraded in a VBSM to increase the vertical angular acceptance of SR light and to enhance sensitivity of the interferometer for measurements of small vertical beam sizes. The larger acceptance of the mirror also eliminated the diffraction peaks observed in $\pi$ polarization method that contributed to a systematic error in our beam size measurements. Subsequently the resolution was observed to be limited in our visible beam size measurement apparatus by transverse beam motion. In order to disentangle effects of the beam motion and beam size and to enable the study of bunch-by-bunch beam dynamics, a fast-gating camera was utilized to measure single bunch transverse beam profiles. We found that the horizontal beam size measured by gated camera is consistently less than those measured by a conventional CCD camera, demonstrating the elimination of turn-by-turn beam motion with single shot capability. In addition, the gated camera enabled the first ever measurement of the width of individual bunches in a train of 30-bunches with $14 \mathrm{~ns}$ spacing. From these measurements we established that the horizontal motion of each bunch increases dramatically along an electron train but not along a positron bunch train under otherwise identical machine condition. The difference in single bunch horizontal dynamics may be a signature for the difference between electron cloud build-up in a train of positron bunches versus the presence of ions for electron bunch trains. We also observed that the effect of the electron cloud is a bunch dependent increase in horizontal as well as vertical emittance.

\section{Acknowledgment}

The authors thank W. Hartung for assistance in one of the measurements, R.E. Meller for setting up timing trigger of the gated camera, and M.G. Billing for valuable discussion. This research was supported by the National Science Foundation under contract numbers PHY-1416318, PHYS1068662, PHYS-1535696, and DMR-1332208.

\section{References}

[1] P. Elleaume, C. Fortgang, C. Penel, E. Tarazona, J. Synchrotron Rad. 2 (1995) 209-214.

[2] B.X. Yang, A.H. Lumpkin, Rev. Sci. Instrum. 67 (1996) 3367-3370.

[3] C.A. Thomas, G. Rehm, DIPAC 2005, Lyon, June 2006, 93-95 (2005).

[4]J. P. Alexander et al., Nucl. Instrum. Methods Phys. Res. A 748 (2014) 96.

[5] T. Mitsuhashi, Proc. of the Joint US-CERN-Japan-Russia School on Particle Accelerators, Montreux, May 1998, 399-427.

[6] T. Mitsuhashi, Proc. of BIW 2004, Knoxville, USA, CP732. 
[7] T. Naito, T. Mitsuhashi, Phys. Rev. ST AB 9 (2006) 122802.

[8] A. Andersson et al., Nucl. Instrum. Methods Phys. Res. A 591 (2008) 437-446.

[9] S.T. Wang et al., IPAC'13, Shanghai, May 2013, p. 849-851 (2013).

[10] W. Cheng et al., PAC'09, Vancouver, June 2009, p. 4015 (2009).

[11] Y. Shoji et al., IBIC'12, Tsukuba, June 2012, p. 302 (2012).

[12] R. Takai et al., DIPAC'11, Hamburg, June 2011, p. 305 (2011).

[13] A.H. Lumpkin et al., EPAC'04, London, June 1994, p. 1687 (1994).

[14] A.S. Fisher et al., SLAC-PUB-11851, 12th BIW, Batavia, IL, May (2006).

[15] J.C. Bergstrom et al., Nucl. Instrum. Methods Phys. Res. A 562, 495 (2006).

[16] J.W. Flanagan et al., EPAC'00, Vienna, June 2000, p. 1119 (2000).

[17] R. Holtzapple, International Workshop on Two Stream Instabilities in Particle Accelerators and Storage Rings, Tsukuba (2001).

[18] R. Holtzapple et al., EPAC'02, Paris, June 2002, p. 410 (2002).

[19] R. Holtzapple et al., PAC'05, Knoxville, May 2005, p. 2006 (2005).

[20] S.T. Wang et al., Nucl. Instrum. Methods Phys. Res. A 703, 80 (2013).

[21] A. Fisher, SLAC-TN-05-048, May (2005).

[22] http://www.hamamatsu.com

[23] http://www.princetoninstruments.com/

[24] J.W. Flanagan et al., EPAC'06, Edinburgh, June 2006, p. 1136-1138. (2006)

[25] M. P. Ehrlichman et al., Phys. Rev. ST Accel. Beams 17 (2014) 044002.

[26] L. Pentecost et al., IPAC'15, Richmond, 2015, p.2390 (2015).

[27] A. Chatterjee et al., Phys. Rev. ST Accel. Beams 18, (2015) 064402. 
a B48W/E

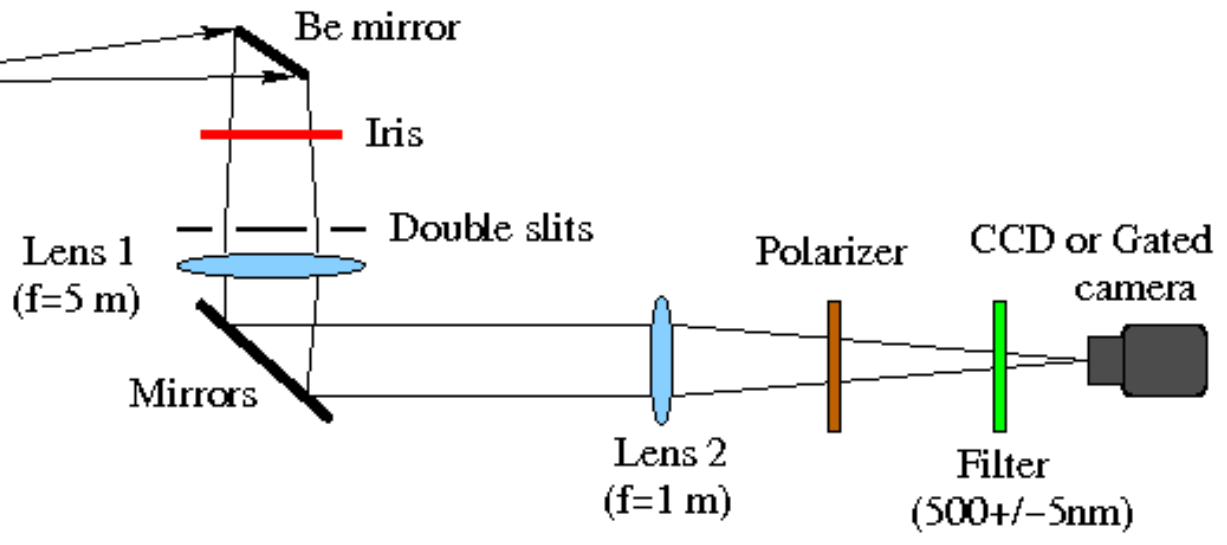

b Polished

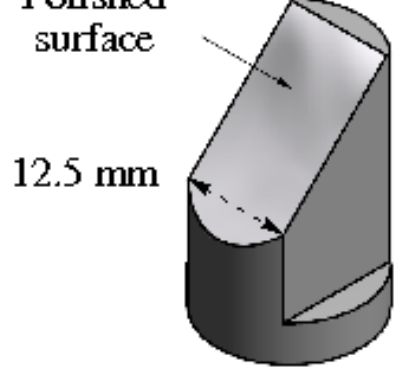

c

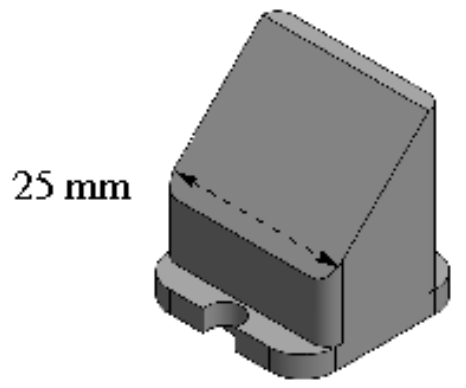

Fig. 1. (a) The schematic setup of visible beam size monitor. The old (b) and new (c) designed Beryllium mirrors. 

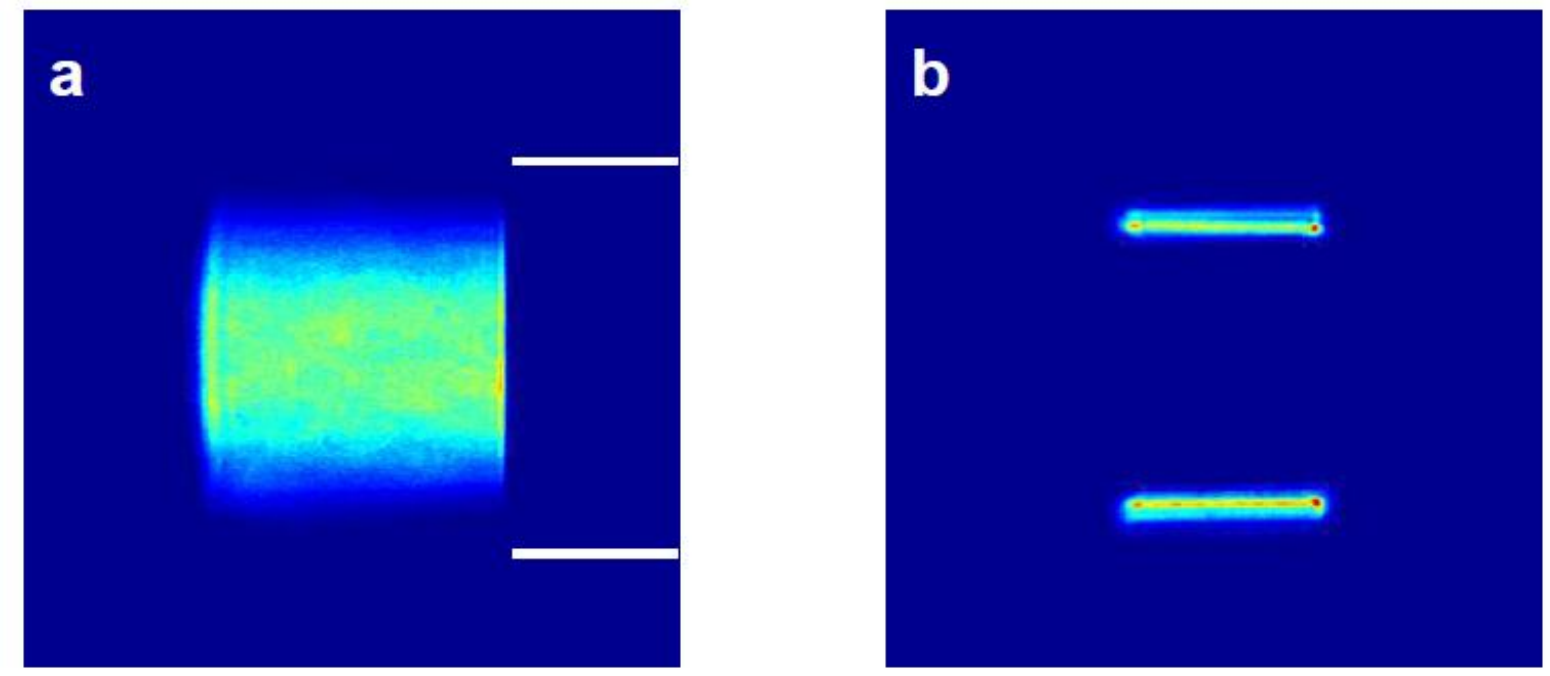

Fig. 2. The CCD images of the new Beryllium mirror (a) and the 14-mm double slit (b). The distance between two white lines in (a) is $20 \mathrm{~mm}$. 

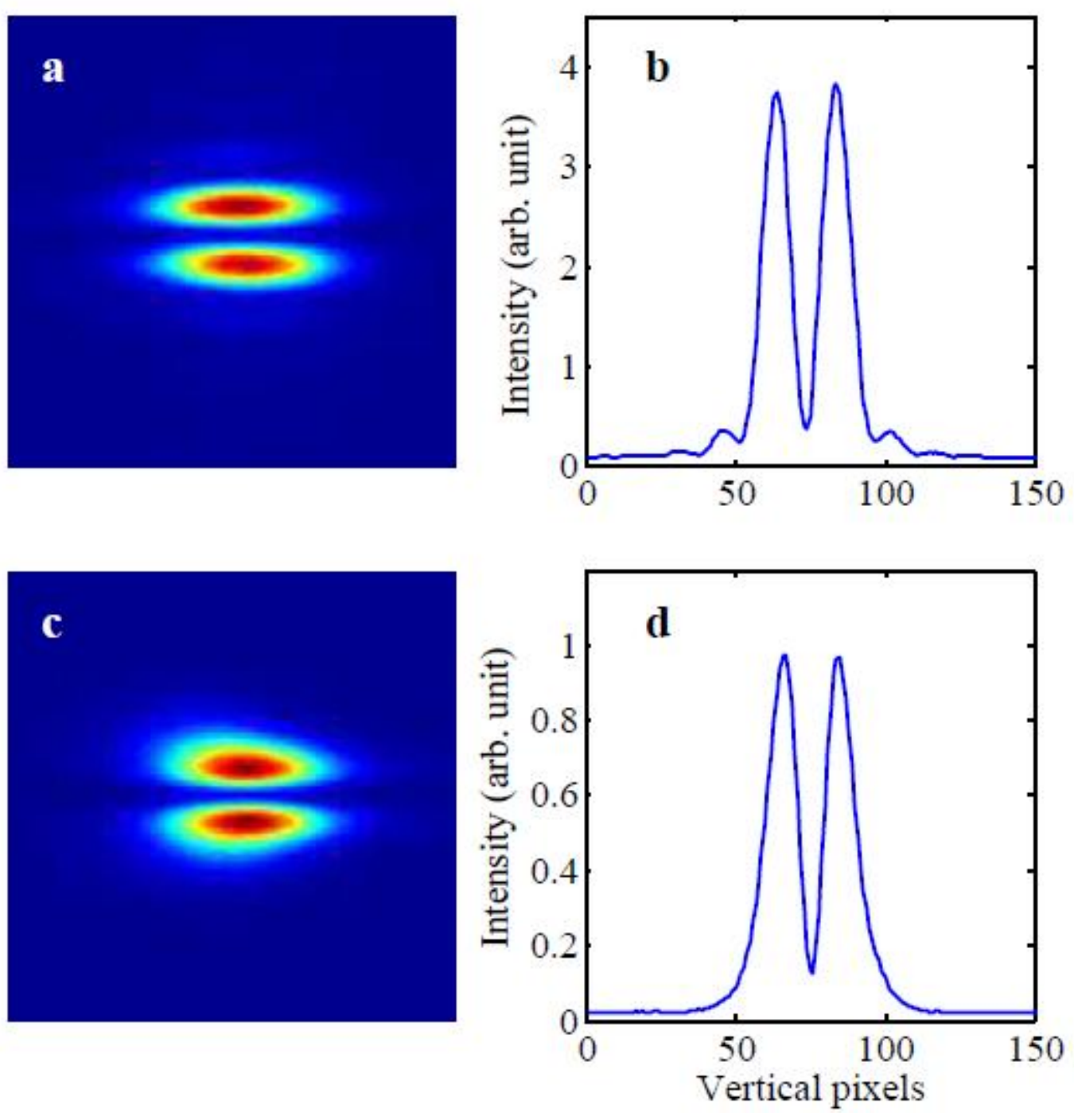

Fig. 3. The measured $\pi$-polarized SR from a single positron bunch at $0.5 \mathrm{~mA}$ using an (a) old and (c) new Be mirror. (b) and (d) are the extracted vertical intensity profile from (a) and (c), respectively. 

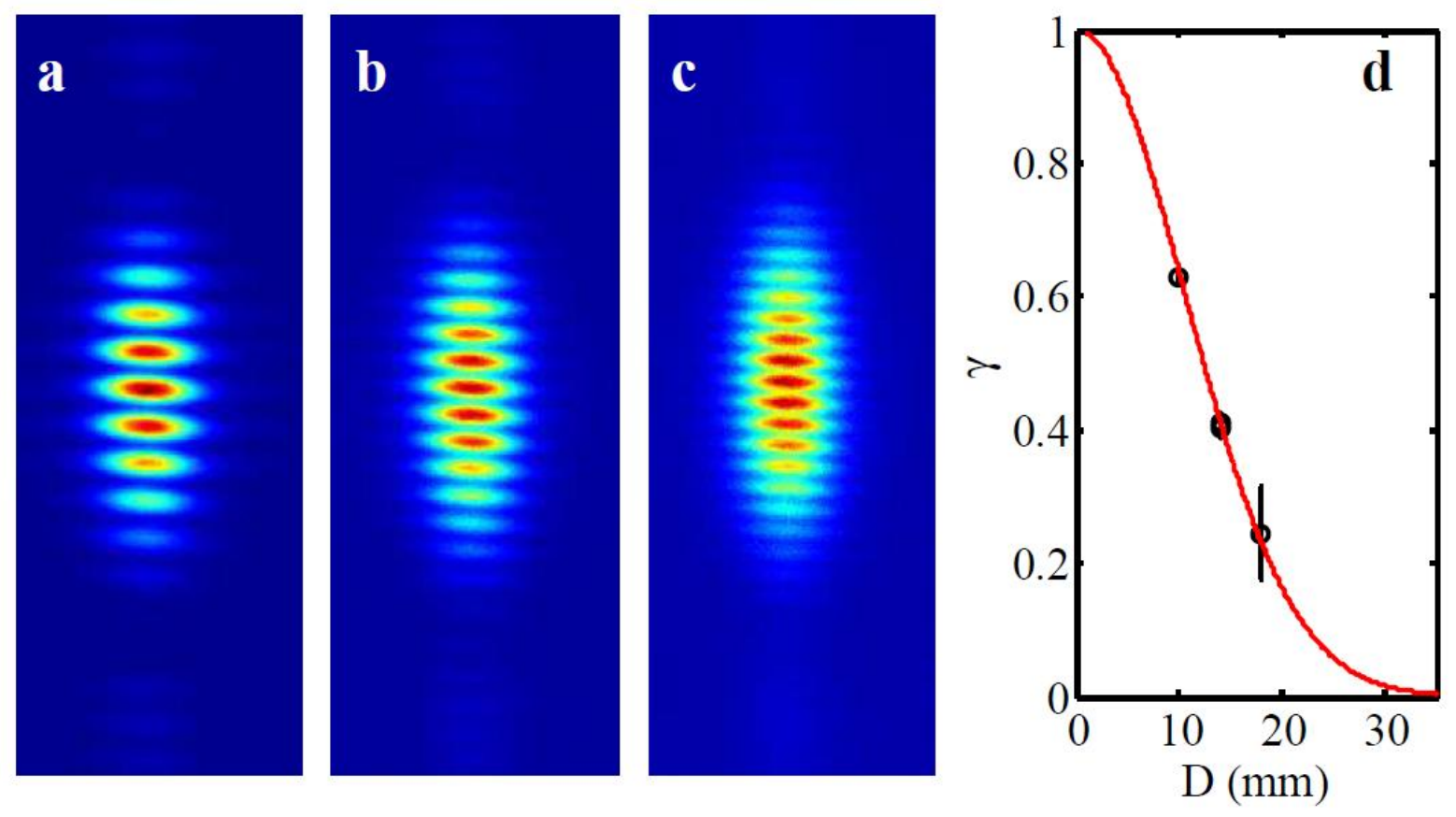

Fig. 4. A vertical beam size measurement using interferometer with three sets of double slits: (a) $\mathrm{D}=10 \mathrm{~mm}$, (b) $14 \mathrm{~mm}$, and (c) $18 \mathrm{~mm}$. After fitting the interference pattern with Eq. (1), the obtained visibility as a function of the double slits separation is plotted in (d). The symbols are data and the line the best fit using Eq. (2). 

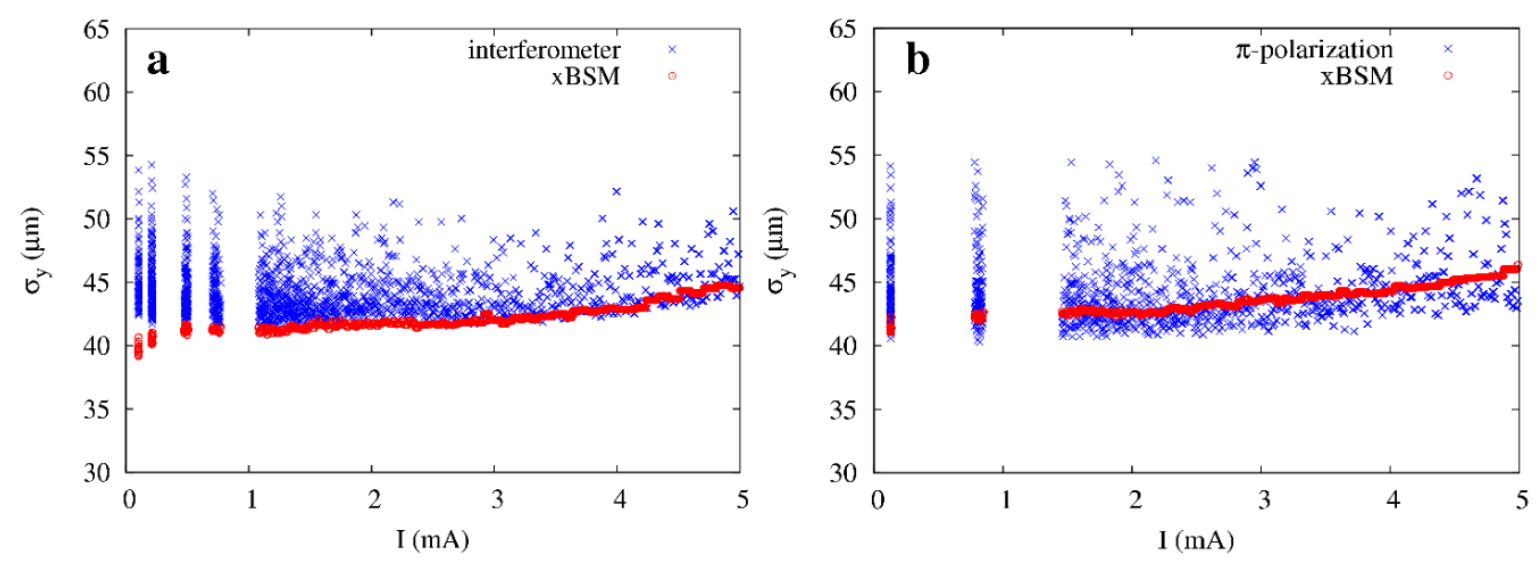

Fig. 5. Measured vertical beam sizes using interferometer (a) and $\pi$-polarization (b) methods as the single electron bunch current decays. A 14-mm double slit was used in the interfereometer measurements. The red circles are the vertical beam sized measured by XBSM. 

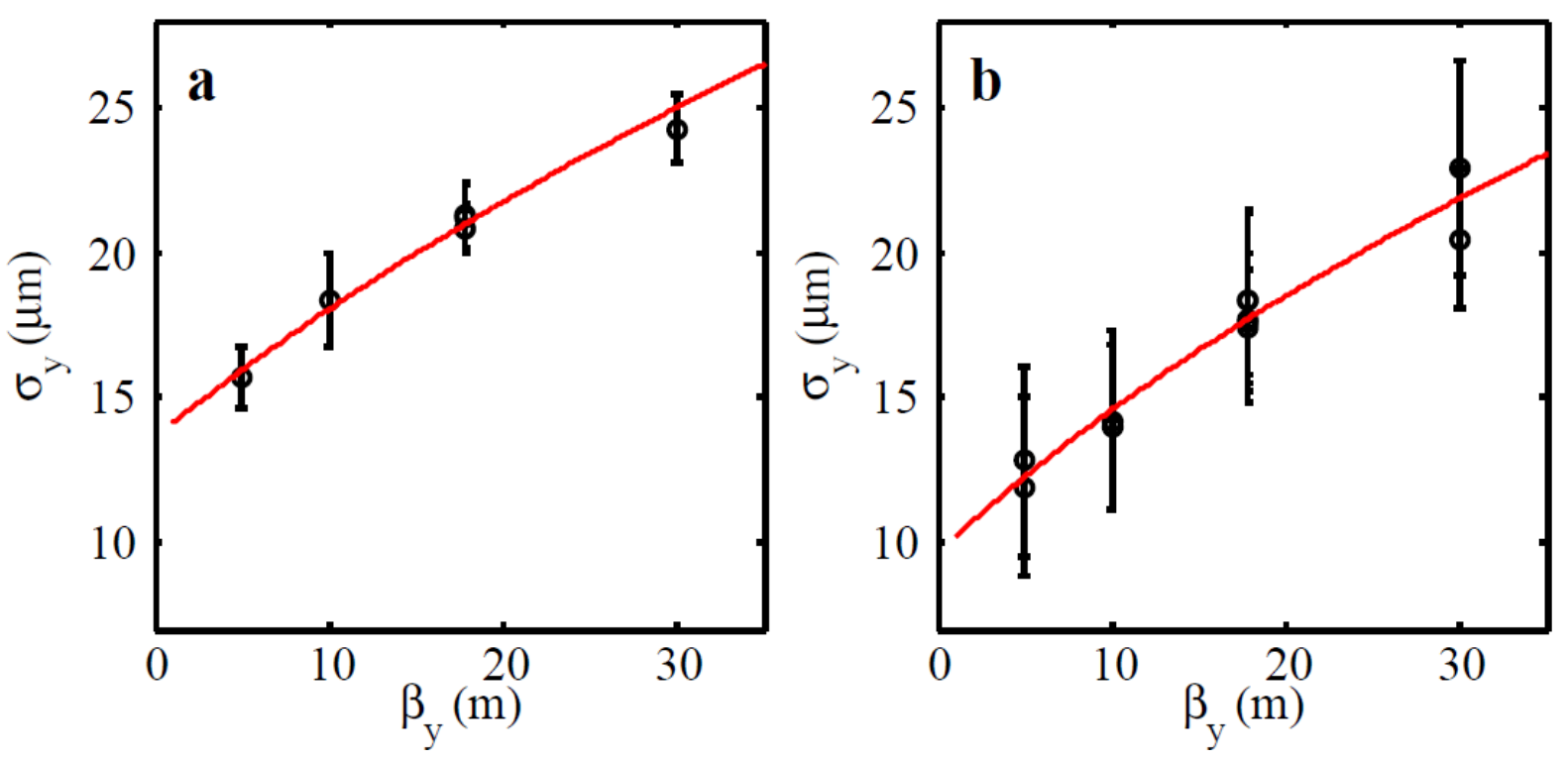

Fig. 6. Vertical beam sizes of a single positron bunch measured with interferometer (a) and $\pi$ polarization (b) methods when $\beta_{y}$ at the vBSM source point changes. The symbols are the data and the red lines are the best fit using Eq. (4). 

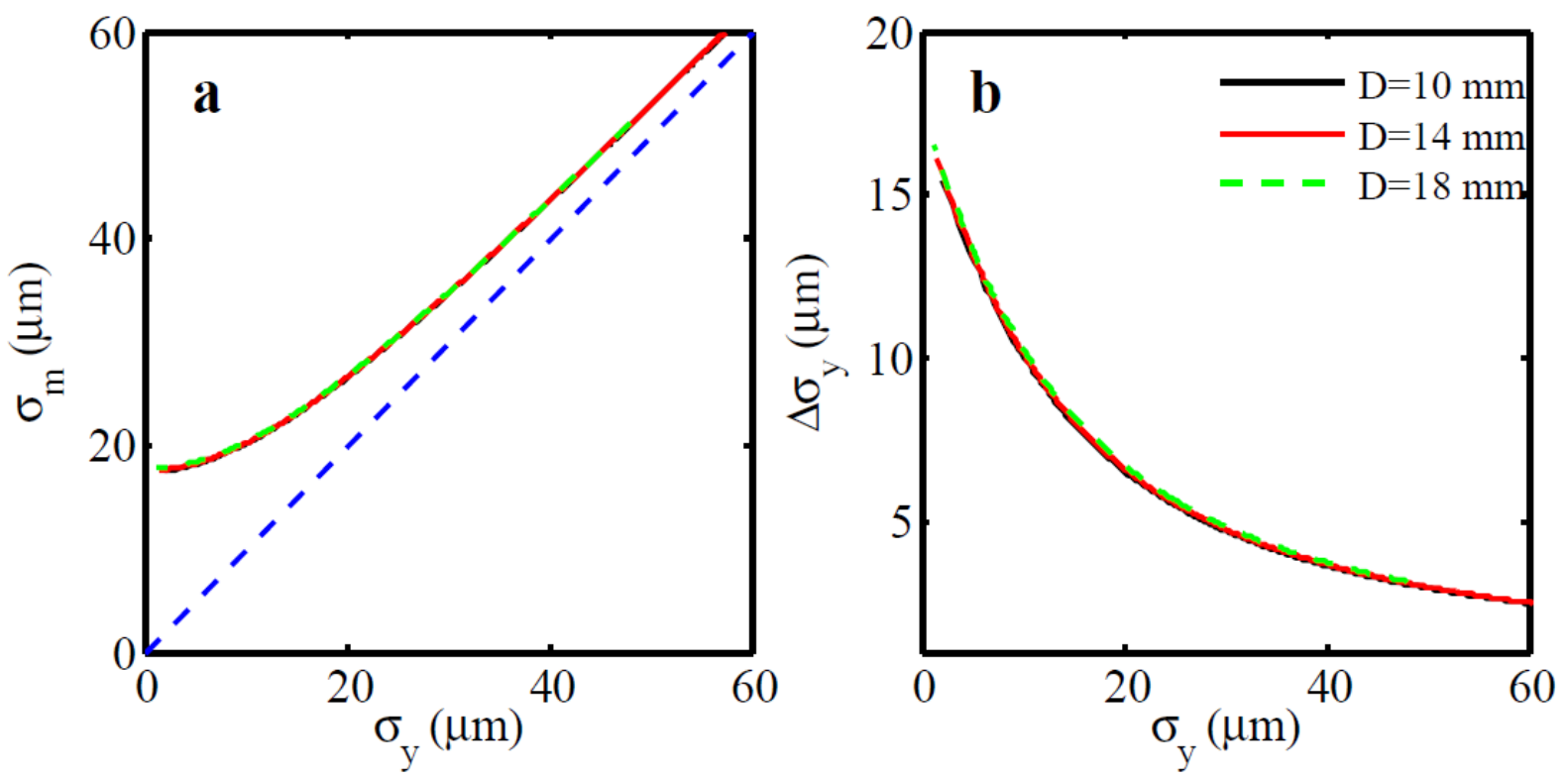

Fig. 7. Assuming $30 \mu \mathrm{m}$ vertical beam motion, $\sigma_{m}$ the expected measured vertical beam size (a) and the beam size error (b) as a function of the actual beam size $\sigma_{y}$ for three different sets of double slits. The blue dashed line in (a) is the guide to the eye. 

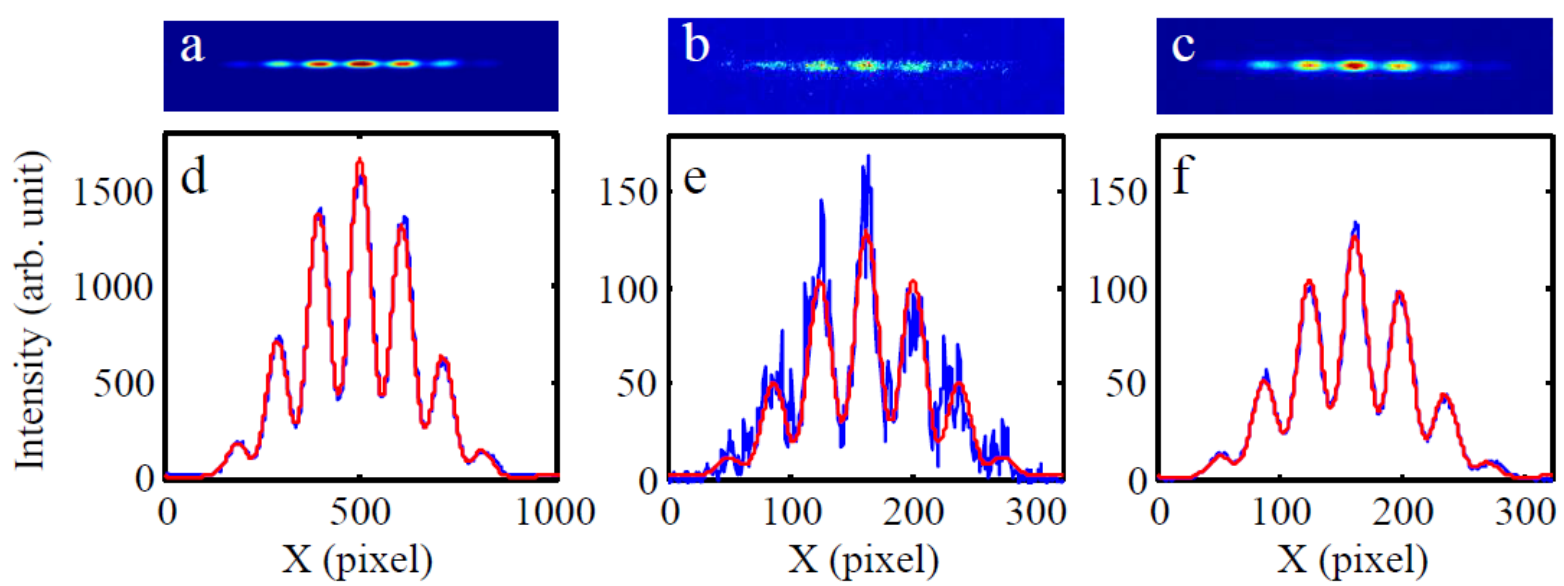

Fig. 8. The interference patterns of a single positron bunch at $0.75 \mathrm{~mA}$ with a $D=2.0 \mathrm{~mm}$ horizontal double slits: (a) from a CCD camera, (b) single image from the gated camera, and (c) averaged image of 100 frames from the gated camera. (d-f) The corresponding interferometer horizontal profiles data (blue) and best fit (red) using Eq. (1). 

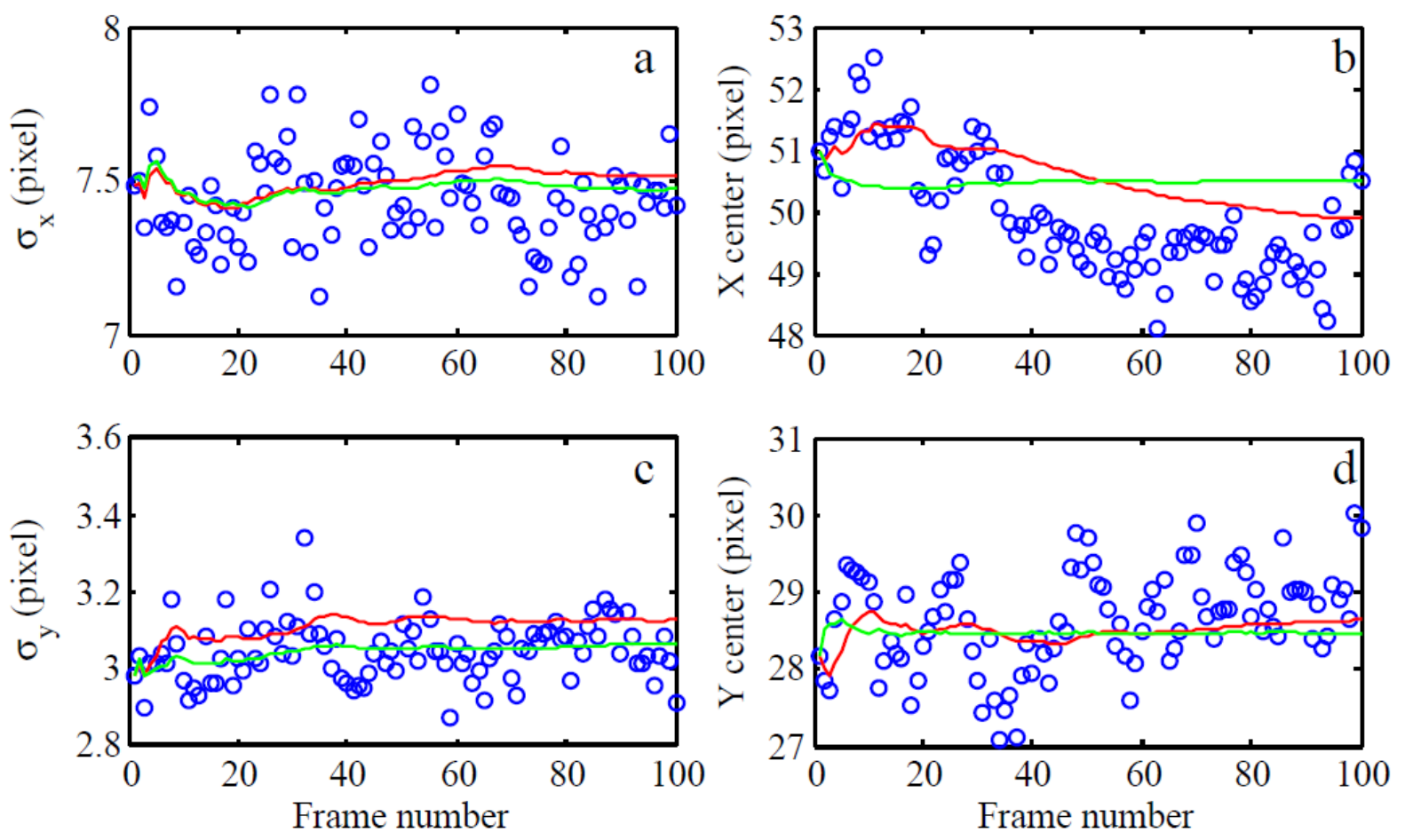

Fig. 9. Comparison between single frame and two averaged methods analysis. (a) and (b) show the horizontal $\sigma_{x}$ and centroid when fitting Gaussian beam files, respectively. (c) and (d) show the vertical $\sigma_{y}$ and centroid. The blue circles are frame by frame Gaussian fit to the data. The red lines are the data from analyzing a direct averaged image. The green lines show the data from analyzing the center-shifted averaged images. 

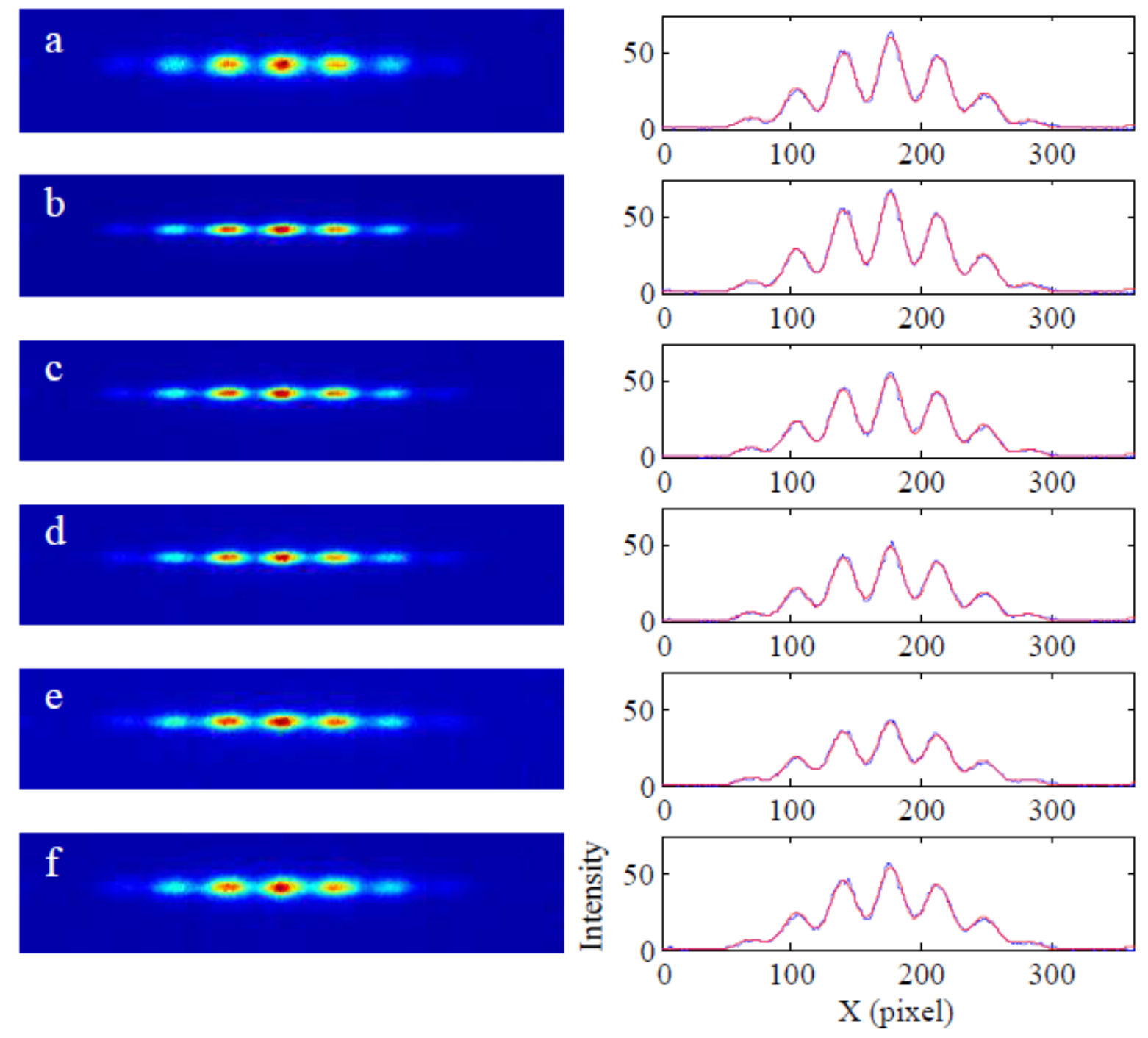

Fig. 10. The measured horizontal interference images of 6 different positron bunches (a) 1, (b) 2, (c) 8 , (d) 16 , (e) 24 , and (f) 30 in a 30 positron bunch train. These images are averaged images over 100 frames captured by gated camera. The right show the corresponding horizontal intensity profiles. 

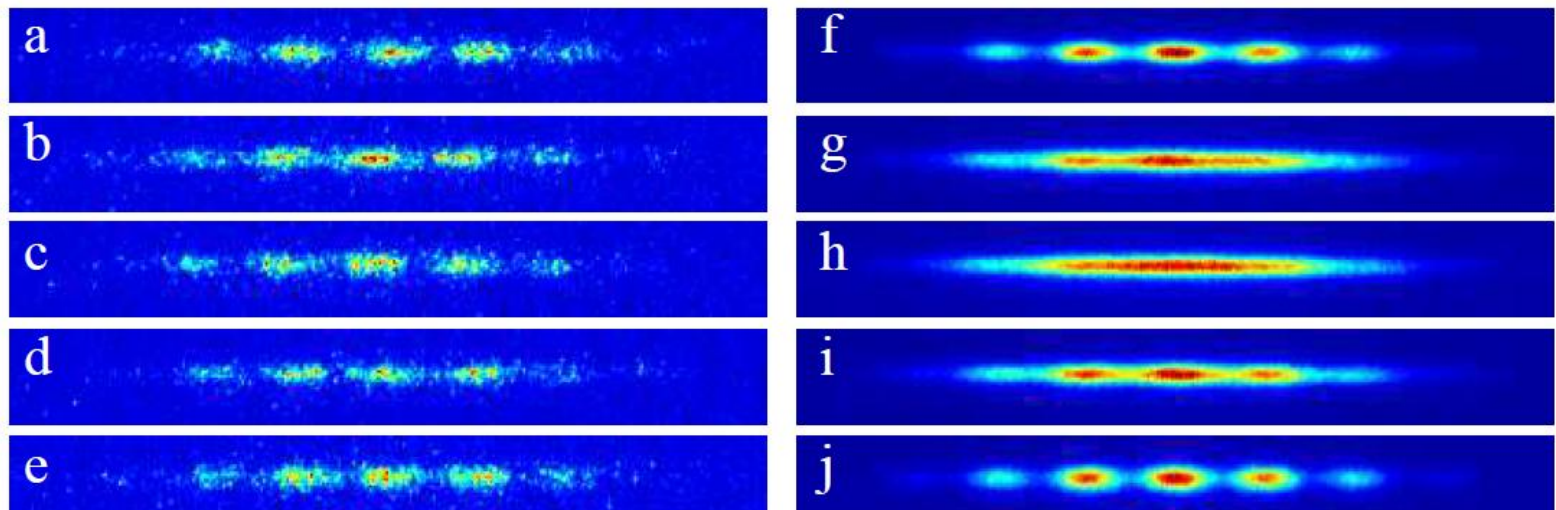

h

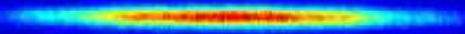

i

$\mathrm{sec}-\mathrm{Con}$

Fig. 11. Single-shot electron interferometer images (a-e) from bunch 1, 1013,16 , and 30 in a 30 electron bunch train. 100 frame average interferometer images ( $f-j)$ of bunch $1,10,13,16$, and 30. 


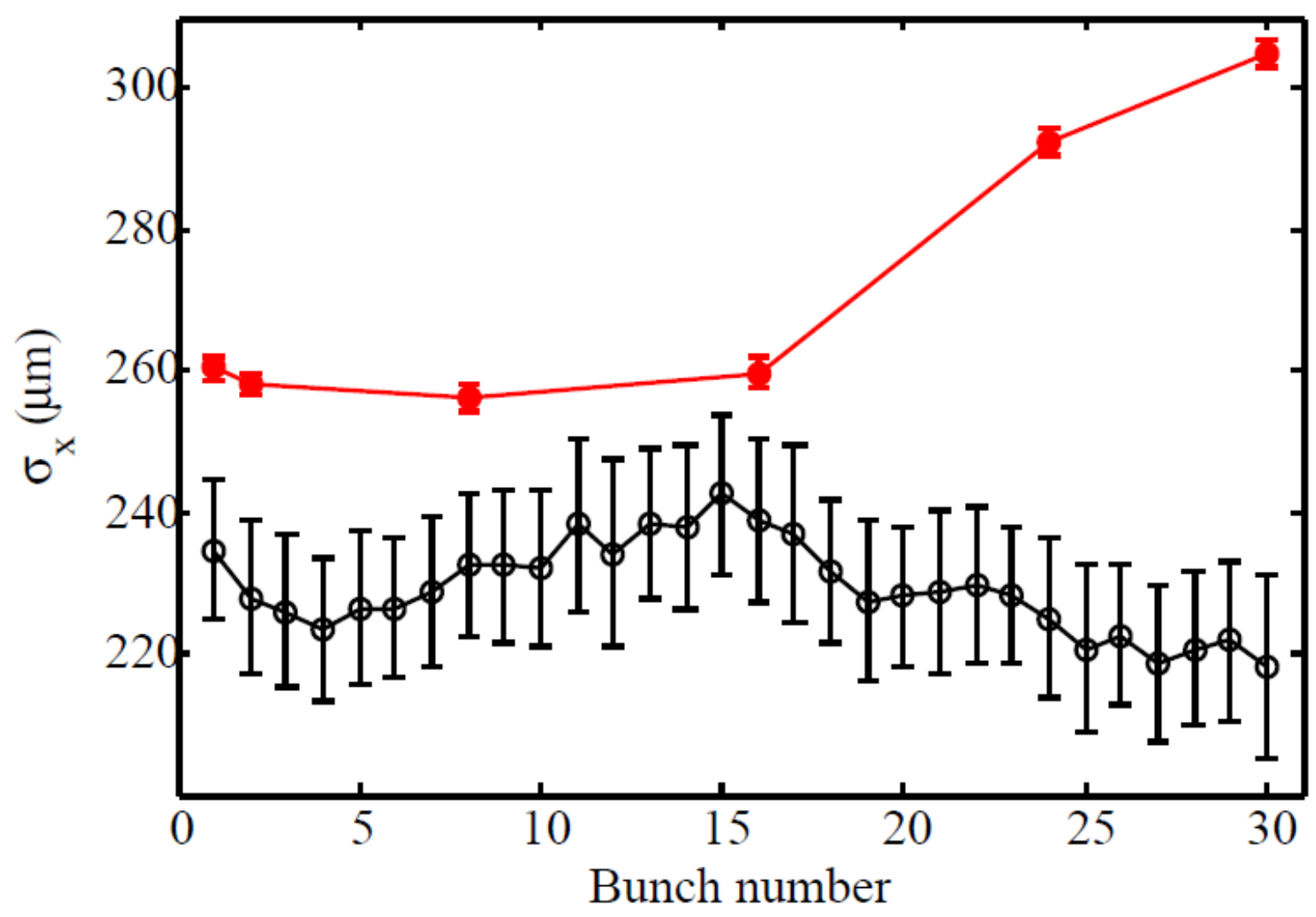

Fig. 12. The horizontal beam sizes along the 30 bunch train of positrons (red) and electrons (black). The beam sizes for positron bunches are analyzed from the averaged interference images while those for electrons are the mean beam sizes from analyzing 100 single shot images. 
Table 1 Beam parameters at VBSM source point in two CESR-TA low emittance energies.

\begin{tabular}{lll}
\hline & Lattice 1 & Lattice 2 \\
\hline Energy $[\mathrm{GeV}]$ & 2.085 & 1.80 \\
\hline$\varepsilon_{\mathrm{x}}[\mathrm{nm}-\mathrm{rad}]$ & 2.6 & 2.5 \\
\hline$\beta_{\mathrm{x}}[\mathrm{m}]$ & 9.2 & 9.5 \\
\hline$\sigma_{\mathrm{x}}[\mu \mathrm{m}]$ & 154 & 155 \\
\hline$\sigma_{\mathrm{E}} / \mathrm{E}[\%]$ & 0.081 & 0.077 \\
\hline$\eta_{\mathrm{x}}[\mathrm{m}]$ & 0.024 & 0.047 \\
\hline$\beta_{\mathrm{y}}[\mathrm{m}]$ & 20.3 & 18.1 \\
\hline$\sigma_{\mathrm{y}}[\mu \mathrm{m}]^{*}$ & 18.6 & 17.5 \\
\hline
\end{tabular}

*Assume the vertical emittance is $17 \mathrm{pm}$-rad. 